\title{
Using Ridge Least Median Squares to Estimate the Parameter by Solving Multicollinearity and Outliers Problems
}

\author{
Kafi Dano Pati ${ }^{1}$, Robiah Adnan ${ }^{1} \&$ Bello Abdulkadir Rasheed ${ }^{1}$ \\ ${ }^{1}$ Department of Mathematical Sciences, Universiti Teknologi Malaysia, 81310 Skudai, Johor, Malaysia \\ Correspondence: Assoc. Prof. Dr. Robiah Adnan, Department of Mathematical Sciences, Faculty of Science, \\ Universiti Teknologi Malaysia, 81310 UTM Johor Bahru, Johor Bahru, Johor, Malaysia. E-mail: \\ robiaha@utm.my
}

Received: October 24, 2014 Accepted: November 1, 2014 Online Published: January 10, 2015

doi:10.5539/mas.v9n2p191 URL: http://dx.doi.org/10.5539/mas.v9n2p191

\begin{abstract}
In the multiple linear regression analysis, the ridge regression estimator is often used to address the problem of multicollinearity. Besides multicollinearity, outliers also constitute a problem in the multiple linear regression analysis. We propose a new biased estimators of the robust ridge regression called the Ridge Least Median Squares (RLMS) estimator in the presence of multicollinearity and outliers. For this purpose, a simulation study is conducted in order to see the difference between the proposed method and the existing methods in terms of their effectiveness measured by the mean square error. In our simulation studies the performance of the proposed method RLMS is examined for different number of observations and the different percentage of outliers. The results of different illustrative cases are presented. This paper also provides the results of the RLMS on a real-life data set. The results show that RLMS is better than the existing methods of Ordinary Least Squares (OLS), Ridge Least Absolute Value (RLAV) and Ridge Regression (RR) in the presence of multicollinearity and outliers.
\end{abstract}

Keywords: multicollinearity, ridge regression, outliers, robust estimation, robust ridge methods

\section{Introduction}

It is clear that the classical ordinary least squares (OLS) estimation has many statistical properties, most of the time when errors are normally distributed the variance of OLS estimation is the least of unbiased estimator. However, OLS estimator is often affected by two problems, namely multicollinearity and outliers which leads to the weakness of OLS estimates (Ma1 and Du1, 2014).

In order to overcome the influence of multicollinearity many biased estimators are introduced. (Hoerl and Kennard, 1970 a, b) First proposed a useful tool called ridge regression for improving prediction in regression situations with highly correlated variables. This method has been successfully applied in many fields. However, the ridge regression method is not robust as it is sensitive to observations that are considered as contaminated data, usually called outliers.

The robust ridge regression estimation has been investigated, to remedy the effect of multicollinearity and outliers. The parameter estimation becomes more difficult when these problems are present in the data set. Different robust estimation methods have been used in the literature to estimate the parameter of regression model in the presence of outliers and multicollinearity. (Pariente, 1977; Askin and Montgomery, 1980; Pfaffenberger and Dielman, 1984; Pfaffenberger, 1985 and Nikolai Krivulin, 1992), among others presented several new ideas concerning the LMS estimator so as to provide some theoretical framework for efficient regression algorithms. (Desire and Leonard, 1986) introduced a procedure to determine the minimum redundancy of a measurement set and the non-redundant samples of measurement that are robust against outliers and multicollinearity.

This paper proposes a new estimation method of a robust ridge estimator that has a high breakdown point called the robust ridge regression estimation method based on least median squares (LMS). The least median squares estimator has $50 \%$ breakdown point as proposed by (Rousseeuw, 1984, 1985), who replaced the least sum of squares (OLS) that has $0 \%$ breakdown point with the least median of squares LMS. (Hampel, 1975), explains the breakdown point as the smallest percentage of contaminated data that can cause the estimator to take a random large abnormal value. 
The aim of evaluating these alternatives biased robust methods is to find the estimator of the parameters of the model that is highly efficient and effective in the presence of multicollinearity and outliers.. The performance of the robust ridge estimators is examined by using the Standard Errors (SE) on a real data set presented by (Hald, 1952). The remainder of the paper is organized as follows; after a brief review of ridge regression in Section 2, we define a robust regression and ridge robust estimators in Section 3. A numerical example is given in section 4, and the results of simulation studies are presented in Section 5. In order to compare the proposed with the existing methods. We shall illustrate that the new estimation method is better than the existing methods when multicollinearity and outliers occur simultaneously. Finally, in section 6 conclusions are presented.

\section{Method}

\subsection{Ridge Regression}

When the least-squares method is applied to non-orthogonal data, we obtain weak estimates of the regression coefficients (Hoerl and Kennard, 1976).

The assumptions of the least squares method that $\hat{\boldsymbol{\beta}}$ is an unbiased estimator of $\boldsymbol{\beta}$. The Gauss-Markov property assures that the least-squares estimator has a minimum variance in the class of unbiased linear estimators in the presence of multicollinearity (Marquardt and Snee, 1975).

One way to reduce this problem is to descend the requirement that the estimator of $\boldsymbol{\beta}$ be unbiased. Assume that a biased estimator of $\boldsymbol{\beta}$ is found to be say $\hat{\boldsymbol{\beta}}$, that has smaller variance than the unbiased estimator. The mean square error of $\beta$ is defined as

$$
\operatorname{MSE}(\hat{\boldsymbol{\beta}})=\operatorname{VAR}(\hat{\boldsymbol{\beta}})+\mathrm{E}(\hat{\boldsymbol{\beta}}-\boldsymbol{\beta})^{2}
$$

Assuming a small amount of bias in $\hat{\boldsymbol{\beta}}$, the variance of $\hat{\boldsymbol{\beta}}$ can be made small such that the Mean Square Error (MSE) of $\hat{\beta}$ is less than the variance of the unbiased estimator $\boldsymbol{\beta}$.

A number of procedures have been employed in obtaining biased estimates of regression coefficients. One of these procedures is ridge regression, primarily proposed by (Hoerl and Kennard, 1970). More specifically, this estimator is defined as the solution to $\hat{\boldsymbol{\beta}}=\left(\mathbf{X}^{\prime} \mathbf{X}+K \mathbf{I}\right)^{-1} \mathbf{X}^{\prime} \mathbf{y}$ where $K \geq 0$ is a constant selected by the analyst. The constant $K$ is usually referred to the biasing parameter, it is obvious that if $K=0$ then the ridge estimator is the least squares estimator. Hence, on the other hand, when $K>0$ the bias $\hat{\boldsymbol{\beta}}_{\text {ridge }}$ increases. However, the variance decreases as $K$ increases.

In this ridge regression, we would like to select a value of $K$ such that the decrease in the variance term is greater than the increase in the squared bias. If this can be done, the mean square error of the ridge estimator $\hat{\boldsymbol{\beta}}_{\text {ridge }}$ will be less than the variance of the least square estimator $\hat{\boldsymbol{\beta}}$.

(Hoerl and Kennard, 1976), proved that there exists a non- zero value of $K$ for which the Mean Square Error (MSE) is less than the variance of the least squares estimator $\hat{\boldsymbol{\beta}}$. We can select a reasonably small value of $K$ at which the ridge estimates $\hat{\boldsymbol{\beta}}_{\text {ridge }}$ are steady. Mostly, this will produce a set of estimates with smaller MSE than the least-squares estimates. (Hoerl and Kennard, 1976), suggested that an appropriate choice of $K$ is where $\hat{\boldsymbol{\beta}}$ and $\hat{\sigma}^{2}$ are found by the least squares solution.

\subsection{Robust Regression Estimators (RRE)}

Ordinary least squares (OLS) regression is very useful, easily interpretable statistical method. However, it is not complete. When we apply an OLS regression, one needs to be aware of its sensitivity to outliers. By "sensitivity to outliers", we mean that an OLS regression model can be highly affected by a few records in the dataset and can then yield results that do not reflect the relationship between the outcome variable and the explanatory variables seen in the rest of the records. Robust regression gives rise to an alternative to OLS regression that is less sensitive to outliers and still defines a linear relationship between the outcome and the explanatory variables. 
There are several different classifications of robust estimates that exist to handle these violations. One important estimator is called the least median squares estimator (Rousseeuw and Leroy, 1987), which has the advantage of minimizing the influence of the residuals. According to (Venables and Ripley, 1999), this algorithm minimizes the median of ordered squares of residuals in order to get the regression coefficients $\boldsymbol{\beta}$ and can be written as equation 2.

Least Median of Squares $=$ Min median

$$
\left|\mathbf{y}-\mathbf{x}_{\mathrm{i}} \boldsymbol{\beta}_{\text {LMS }}\right|^{2}
$$

(Martin, 2002) describes the median squared residuals lack a smooth squared residual function and the asymptotic rate is $n^{1 / 3}$ to converge efficiently under normality it means that LMS converges less smoothly and weaker than the LTS algorithm. And also, takes a long time to converge. Least Median of Squares (LMS) estimator is one of the true high breakdown point estimators that reached the above mentioned upper boundary of the breakdown point. (Rousseeuw, 1984).

\subsubsection{Robust Ridge Regression Estimators (RRRE)}

(Pfaffenberger and Dielman 1985, 1990), proposed robust ridge regression by extending the development of their technique by performing Monte Carlo simulation studies to compare various approaches. The proposed method in this paper combines the LMS estimator with the ridge regression estimator which is referred to as the RLMS estimator. So, RLMS robust ridge estimators will be resistant to multicollinearity problems and less affected by outliers. The RLMS estimator can be written as equation 3 .

$$
\hat{\boldsymbol{\beta}}_{\mathrm{RLMS}}=\left(\mathbf{X}^{\prime} \mathbf{X}+K_{\mathrm{LMS}} \mathbf{I}\right)^{-1} \mathbf{X}^{\prime} \mathbf{Y}
$$

The value of $K$ is determined from data using equation 4 .

$$
K_{\mathrm{LMS}}=\frac{p S_{\mathrm{LMS}}^{2}}{\hat{\boldsymbol{\beta}}_{\mathrm{LMS}}^{\prime} \hat{\boldsymbol{\beta}}_{\mathrm{LMS}}} \text { and } S_{\mathrm{LMS}}^{2}=\frac{\sum_{i=1}^{n} \varepsilon_{\mathrm{LMS}}^{2}}{n-p}
$$

where $p$ is the number of independent variables, $n$ is the number of observations in the data, $\hat{\boldsymbol{\beta}}_{\mathrm{LMS}}$ is the estimates of $\boldsymbol{\beta}$ and $\varepsilon_{\text {LMS }}^{2}$ is the residuals from LMS method.

\section{Results}

\subsection{Numerical Result}

A real data set of (Hald, 1952) is considered to assess the effectiveness of the proposed robust ridge regression method. This data set consists of 4 variables and 13 observations with 3 outliers. The response variable (y) is the heat evolved from a particular mixture of cement, and the covariates are tricalcium aluminate (x1), tricalcium silicate (x2), tetracalcium alumina ferrite (x3), and dicalcium silicate (x4).

(Table 1) contains the parameters' estimates, the standard error of the parameters' estimates and Variance Inflation Factor (VIF) analysis of the Hald Data. The VIF for each of these predictors are all extremely high, indicating troublesome multicollinearity with the presence of outliers in the data, the use of robust method provides more stable parameter estimates.

With this aim, initial robust regression estimates was first calculated to obtain robust ridge estimates in the presence of both multicollinearity and outliers; these estimates are given in (Table 1)

Table 1. Estimated parameter and SE of $\hat{\boldsymbol{\beta}}_{1}, \hat{\boldsymbol{\beta}}_{2}, \hat{\boldsymbol{\beta}}_{3}$ and $\hat{\boldsymbol{\beta}}_{4}$ for the different methods with the variance inflation factor.

\begin{tabular}{ccccccc}
\hline Coef. & Estimate & \multirow{2}{*}{ VIF } & OLS & RIDGE & RLMS & RLAV \\
\hline$\hat{\boldsymbol{\beta}}_{1}$ & parameter & \multirow{2}{*}{38.4962} & 1.5511 & 0.5081 & 0.3964 & 0.6466 \\
& s.e. & & 0.7448 & 0.0791 & 0.1036 & 0.1325 \\
$\hat{\boldsymbol{\beta}}_{2}$ & parameter & \multirow{2}{*}{254.423} & 0.5102 & 0.3102 & 0.3545 & 0.6082 \\
& s.e. & & 0.7238 & 0.0916 & 0.1070 & 0.2726 \\
$\hat{\boldsymbol{\beta}}_{3}$ & parameter & \multirow{2}{*}{46.8684} & 0.1019 & -0.0605 & -0.0480 & 0.1253 \\
$\hat{\boldsymbol{\beta}}_{4}$ & p.e. & & 0.7547 & 0.0777 & 0.1012 & 0.1392 \\
& parameter & \multirow{2}{*}{282.513} & -0.1441 & -0.3879 & -0.3548 & -0.0859 \\
& & & 0.7091 & 0.0925 & 0.1064 & 0.2856 \\
\hline
\end{tabular}


The standard error of the proposed RLMS method is lower than the existing RLAV in the presence of outliers. (Table 1) shows that the data have a high VIF for all variables with three outliers.

Also, the standard errors of the parameters'estimates for RLMS are less than all except for the ridge regression whose difference is smaller.

\subsection{Simulation Study}

We carry out a simulation study to compare the performance of the different methods OLS, RR and RLAV with the proposed estimator RLMS. The simulation is designed to allow both multicollinearity and non-normality to exist simultaneously. The non-normal distributions are used to generate outliers.

Suppose, we have the following linear regression model. (Siti Meriam et al., 2012)

$$
\mathbf{y}_{\mathbf{i}}=\boldsymbol{\beta}_{\mathbf{0}}+\boldsymbol{\beta}_{1} \mathbf{x}_{\mathbf{i} 1}+\boldsymbol{\beta}_{\mathbf{2}} \mathbf{x}_{\mathbf{i} 2}+\boldsymbol{\beta}_{3} \mathbf{x}_{\mathbf{i} 3}+\mathbf{e}_{\mathbf{i}}, \text { where } \mathrm{i}=1,2,3
$$

The parameter values $\boldsymbol{\beta}_{\mathbf{0}}, \boldsymbol{\beta}_{\mathbf{1}}, \boldsymbol{\beta}_{\mathbf{2}}$ and $\boldsymbol{\beta}_{\mathbf{3}}$ are set equal to one. The explanatory variables $\mathbf{x}_{\mathbf{i} 1}, \mathbf{x}_{\mathbf{i} 2}$ and $\mathbf{x}_{\mathbf{i} 3}$ are generated using equation (6)

$$
x_{i j}=\left(1-\rho^{2}\right)+\rho z_{i j} \quad \mathrm{i}=1,2, \ldots, \mathrm{n}, \mathrm{j}=1,2,3
$$

where, $z_{i j}$ are independent standard normal random numbers generated by the normal distribution.

The explanatory variables' values were generated for a given sample size $\mathrm{n}$. The sample sizes used were 50 and 100. The value of $\rho$ representing the correlation between any two explanatory variables, and its values were chosen as: $0.0,0.5$ and 0.99 . The percentage of outliers present in this data set is $20 \%$.

The number of replications used is 500. The statistics computed are the bias, root of mean squared error (RMSE), standard error (SE), and 6 pairwise MSE ratios of the estimators.

The bias and MSE are given as: Bias $=\bar{\beta}_{i}-\beta_{i}$ where $\bar{\beta}_{i}=\frac{\sum_{i=1}^{k} \beta_{i}}{k} k=500$, and the mean squared error (MSE) is $M S E=\frac{1}{500} \sum_{i=1}^{500}\left(\hat{\boldsymbol{\beta}}_{\mathbf{i}}-\boldsymbol{\beta}_{\mathbf{i}}\right)^{2}$, therefore, the RMSE is given by

$$
\left[\operatorname{MSE}\left(\hat{\boldsymbol{\beta}}_{\mathbf{j}}\right)\right]^{1 / 2} \quad \text { where } \mathbf{j}=0,1,2,3
$$

The VIF for the simulated data are shown in (Table 2)

Table 2. The VIF for the simulated data

\begin{tabular}{lccc}
\hline \multicolumn{4}{c}{$\rho=0.99$} \\
\hline Var & X1 & X2 & X3 \\
\hline VIF (N=50) & 126.1541 & 204.3971 & 112.0508 \\
VIF (N=100) & 133.8203 & 238.6387 & 141.2589 \\
\hline
\end{tabular}

Here the maximum VIF is 238.6387 when the correlation between independent variables was very high with different sample size. So it is clear that the multicollinearity problem exists.

The measure of convergence was computed as the number of times estimator 2 or 3 to the true parameter while the value in (Table 3 and Table 4) show the summary statistics such as bias, root mean squared error (RMSE) and standard error (SE) of the estimators of the normal distributions for sample size 50 and 100 with $0 \%$ and $20 \%$ of outliers and different value of $\rho$.

On the other hand, when we apply these methods to the simulated data with different sample size in the presence of different percentage of outliers and different levels of multicollineaity, we obtained that the standard errors for proposed method are less than the standard errors of the aforementioned existing methods. 
(Table 5) shows the efficiency of the estimators by looking at the MSE ratios of the estimators written as follows.

$$
\text { MSE }_{\text {ratios }}=\frac{\text { RMSE (proposed method) }}{\text { RMSE (existing method) }}
$$

This Table supported the result obtained from (Table 3 and Table 4). These ratios show the efficiency of RLMS relative to other estimators then $\mathrm{MSE}_{\text {ratios }}$ less than 1 denotes that the estimator is more efficient, however, values more than 1 denote that the other estimator is more efficient.

From (Table 3 and Table 4) we can see that the RMSE of the OLS is relatively smaller than the other estimators when the errors are normally distributed that is, without multicollinearity and no outliers.

As expected, the OLS gives the best result in the ideal situation. Also, the result in (Table 5) is in favor of LS. However, we see in (Table 5) that the ratio of MSE of RLMS to OLS is greater than 1.00 denoting that the OLS is more efficient than the RLMS when no multicollinearity and no outliers.

On the other hand, we can see from the (Table 3 and Table 4) that the RMSE of the RIDGE is relatively smaller than the RLAV. Also, the values of MSE ratios for the estimators ridge regression are less than 1 indicates that the estimator is less efficient than RLAV and RLMS when the errors are normally distributed without outliers and no multicollinearity. While, for non-normal error distribution and when correlation and outliers are present in the data, RLMS is better than the OLS, RIDGE and RLAV. RLAV is almost as good as RIDGE and OLS.

Consequently, we can see that the RMSE of the RLMS is relatively smaller than the other estimators when the errors are normally distributed in the presence of multicollinearity and outliers. It obviously shows that RLMS is more efficient than RLAV and RIDGE but certainly much more efficient than OLS when multicollinearity and outliers are present.

Table 3. Bias, RMSE and SE of $\hat{\boldsymbol{\beta}}_{1}, \hat{\boldsymbol{\beta}}_{2}$ and $\hat{\boldsymbol{\beta}}_{3}$ with error normal $(0,1)$ distribution of the sample size $\mathrm{n}=50$ correlation $0.0,0.5,0.99$ outliers $0 \%$ and $20 \%$

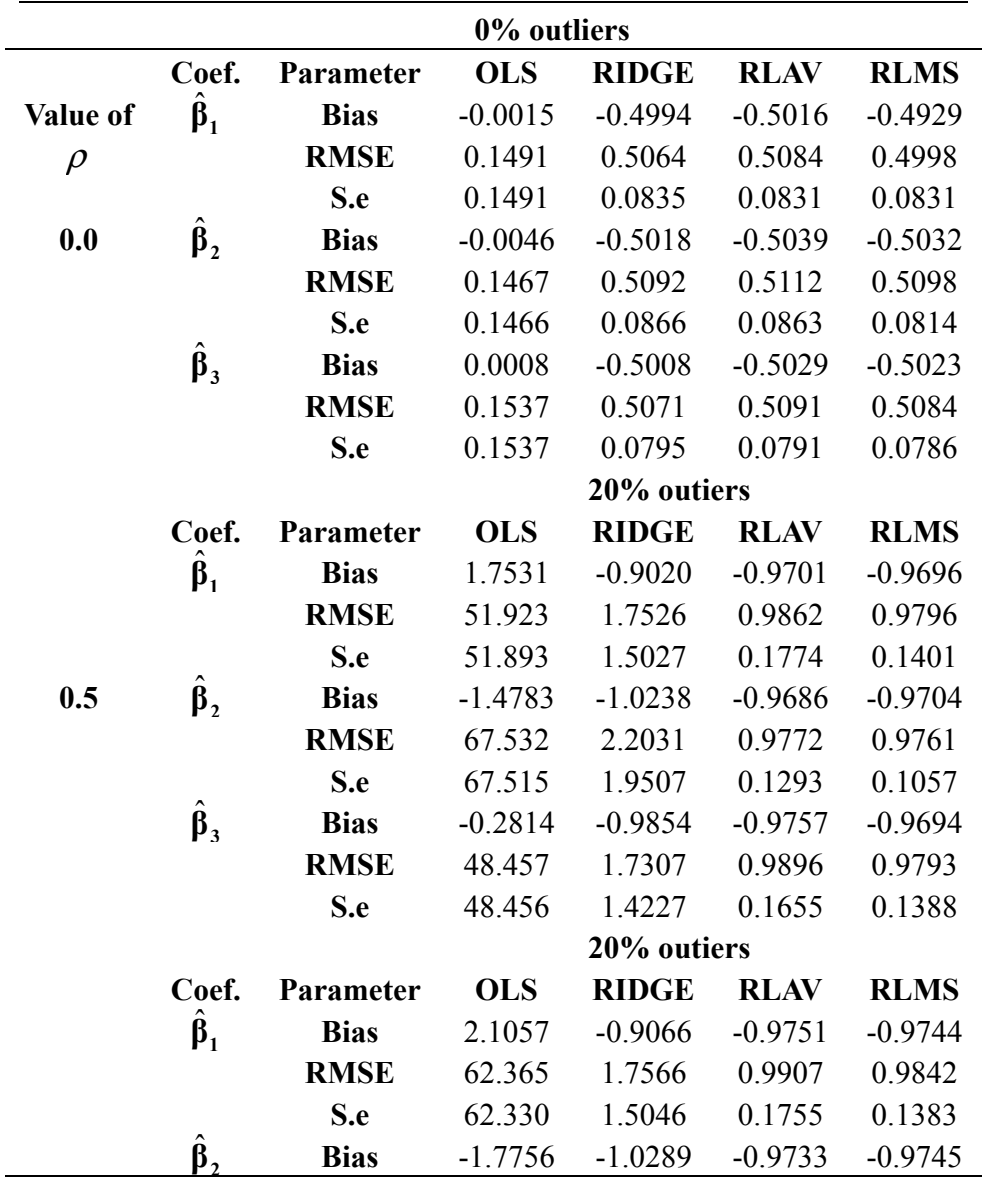




\begin{tabular}{ccccccc}
\hline $\mathbf{0 . 9 9}$ & & RMSE & 81.113 & 2.2077 & 0.9817 & 0.9800 \\
& & S.e & 81.094 & 1.9533 & 0.1281 & 0.1045 \\
& & Bias & -0.3379 & -0.9904 & -0.9804 & -0.9741 \\
& $\hat{\boldsymbol{\beta}}_{3}$ & RMSE & 58.203 & 1.7349 & 0.9940 & 0.9837 \\
& & S.e & 58.202 & 1.4245 & 0.1638 & 0.1370 \\
\hline
\end{tabular}

Table 4. Bias, RMSE and SE of $\hat{\boldsymbol{\beta}}_{1}, \hat{\boldsymbol{\beta}}_{2}$ and $\hat{\boldsymbol{\beta}}_{3}$ with error normal $(0,1)$ distribution of the sample size $\mathrm{n}=100$ correlation $0.0,0.5,0.99$ outliers $0 \%$ and $20 \%$

\begin{tabular}{|c|c|c|c|c|c|c|}
\hline \multirow{5}{*}{$\begin{array}{c}\text { Value of } \\
\quad \rho\end{array}$} & \multicolumn{6}{|c|}{$0 \%$ outliers } \\
\hline & Coef. & Parameter & OLS & RIDGE & RLAV & RLMS \\
\hline & \multirow{3}{*}{$\hat{\boldsymbol{\beta}}_{1}$} & Bias & 0.0055 & -0.4987 & -0.4991 & -0.4984 \\
\hline & & RMSE & 0.1032 & 0.5018 & 0.5022 & 0.5015 \\
\hline & & S.e & 0.1031 & 0.0562 & 0.0561 & 0.0557 \\
\hline \multirow[t]{11}{*}{0.0} & \multirow{4}{*}{$\hat{\boldsymbol{\beta}}_{2}$} & Bias & 0.0146 & -0.496 & -0.4964 & -0.4951 \\
\hline & & RMSE & 0.1078 & 0.4991 & 0.4995 & 0.4982 \\
\hline & & S.e & 0.1068 & 0.0557 & 0.0556 & 0.0551 \\
\hline & & Bias & -0.0040 & -0.5003 & -0.5007 & -0.4953 \\
\hline & \multirow{3}{*}{$\hat{\boldsymbol{\beta}}_{3}$} & RMSE & 0.1023 & 0.5039 & 0.5043 & 0.4988 \\
\hline & & $\sigma$ & 0.1022 & 0.0604 & 0.0603 & 0.0596 \\
\hline & & & \multicolumn{3}{|c|}{$20 \%$ outiers } & \\
\hline & Coef. & Parameter & OLS & RIDGE & RLAV & RLMS \\
\hline & & Bias & -1.5448 & -1.0143 & -0.9750 & -0.9695 \\
\hline & $\hat{\boldsymbol{\beta}}_{1}$ & RMSE & 33.827 & 1.4107 & 0.9969 & 0.9856 \\
\hline & & S.e & 33.7918 & 0.9806 & 0.2077 & 0.1774 \\
\hline \multirow[t]{11}{*}{0.5} & \multirow{4}{*}{$\hat{\boldsymbol{\beta}}_{2}$} & Bias & 3.2966 & -0.8810 & -0.9654 & -0.9651 \\
\hline & & RMSE & 48.607 & 1.6624 & 0.9812 & 0.9776 \\
\hline & & Se & 48.495 & 1.4098 & 0.1752 & 0.1559 \\
\hline & & Bias & -1.8926 & -1.0194 & -0.9753 & -0.9683 \\
\hline & \multirow{3}{*}{$\hat{\boldsymbol{\beta}}_{3}$} & RMSE & 32.248 & 1.3915 & 0.9941 & 0.9851 \\
\hline & & S.e & 32.193 & 0.9472 & 0.1923 & 0.1810 \\
\hline & & & \multicolumn{3}{|c|}{$20 \%$ outiers } & \\
\hline & Coef. & Parameter & OLS & RIDGE & RLAV & RLMS \\
\hline & & Bias & -1.8554 & -1.0192 & -0.9798 & -0.9743 \\
\hline & & RMSE & 40.630 & 1.4151 & 1.0008 & 0.9895 \\
\hline & $\mathbf{p}_{1}$ & S.e & 40.588 & 0.9816 & 0.2041 & 0.1725 \\
\hline \multirow[t]{6}{*}{0.99} & & Bias & 3.9596 & -0.8858 & -0.9706 & -0.9703 \\
\hline & $\hat{\boldsymbol{\beta}}_{2}$ & RMSE & 58.383 & 1.6661 & 0.9857 & 0.9822 \\
\hline & & S.e & 58.2486 & 1.4112 & 0.1719 & 0.1522 \\
\hline & & Bias & -2.2732 & -1.024 & -0.9800 & -0.9820 \\
\hline & $\hat{\boldsymbol{\beta}}_{3}$ & RMSE & 38.734 & 1.3959 & 0.9980 & 0.9977 \\
\hline & & S.e & 38.667 & 0.9482 & 0.1886 & 0.1759 \\
\hline
\end{tabular}

Table 5. MSE ratios of 6 pairwise estimators of $\hat{\boldsymbol{\beta}}_{1}, \hat{\boldsymbol{\beta}}_{2}, \hat{\boldsymbol{\beta}}_{3}$ with errors normal $(0,1)$ distribution and $0 \%$ and $20 \%$ of outliers

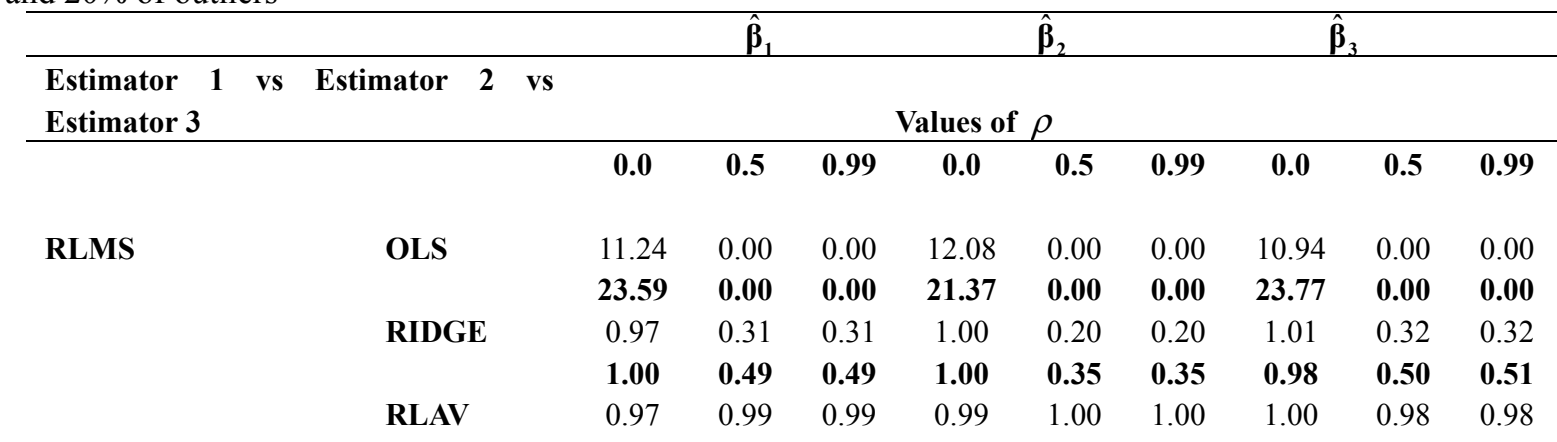




\begin{tabular}{|c|c|c|c|c|c|c|c|c|c|c|}
\hline & & 1.00 & 0.98 & 0.98 & 0.99 & 0.99 & 0.99 & 0.98 & 0.98 & 1.00 \\
\hline \multirow[t]{4}{*}{ RLAV } & OLS & 11.63 & 0.00 & 0.00 & 12.15 & 0.00 & 0.00 & 10.97 & 0.00 & 0.00 \\
\hline & & 23.66 & 0.00 & 0.00 & 21.48 & 0.00 & 0.00 & 24.30 & 0.00 & 0.00 \\
\hline & RIDGE & 1.01 & 0.32 & 0.32 & 1.01 & 0.20 & 0.20 & 1.01 & 0.33 & 0.33 \\
\hline & & 1.00 & 0.50 & 0.50 & 1.00 & 0.35 & 0.35 & 1.02 & 0.51 & 0.51 \\
\hline \multirow[t]{2}{*}{ RIDGE } & OLS & 11.53 & 0.00 & 0.00 & 12.05 & 0.00 & 0.00 & 10.89 & 0.00 & 0.00 \\
\hline & & 23.62 & 0.00 & 0.00 & 21.45 & 0.00 & 0.00 & 24.26 & 0.00 & 0.00 \\
\hline
\end{tabular}

The simulation results for larger samples, that is for $n=100$ are consistent with the results of the smaller sized samples. The results also show that the estimator for larger samples are more efficient than those of smaller samples since the values of RMSE are smaller.

\section{Discussion}

Multicollinearity data sets with outliers are very common in real life situation. In order to remedy both problems, robust biased estimation methods are applied. The best model is chosen by looking at the RMSE value.

The simulation study in section 5 provides' RMSE values, bias and standard errors of the OLS, ridge, RLAV and RLMS estimators. It can be observed that the RMSE obtained from RLMS is the minimum.

Thus, RLMS is the best method. When there is no multicollinearity and outliers, ridge regression has the least RMSE value, thus the best method. However, when there is multicollinearity and outliers in the data, then RLMS has the least RMSE value, thus it is considered the best method.

We also use a real-life data set to study the effect of multicollinearity and outliers. The results obtained from our proposed method of RLMS are better than the OLS, Ridge and RLAV in terms of their RMSE values. Consequently, in this study, it is shown that the results obtained from the numerical data set and a simulation study with both multicollinearity and outliers, RLMS gives better results followed by RLAV method. This result is true for $\rho=0.0,0.5$ and 0.99 with the sample size of $\mathrm{n}=50$ and 100 .

\section{Acknowledgements}

This work was supported by the Universiti Teknologi Malaysia, under the Research University Grant (QJ130000.2526.6 H68) and Ministry of Higher Education (MOHE) of Malayisa.

\section{References}

Askin, R. G., \& Montgomery, D. C. (1980). Augmented robust estimators technometrics, 22, 333-341. Insightful Corp Presentation, New York. 17-18.

Desire, L. M., \& Leonard, K. (1986). A robust method for outlier and model error detection in regression and calibration. Analytica chimica Acta., 187, 171-179. http://dx.doi.org/10.1016/S0003-2670(00)82910-4

Haled. (1952). Statistical Theory with Engineering Application. Wiley, New York. p. 647.

Hampel, F. R. (1975). Beyond location parameters: Robust concepts and methods proceedings of the $40^{\text {th }}$ session of the ISI., 46(1), 375-391.

Hoerl, A. E., \& Baldwin, K. F. (1976). Ridge Regression: Some Simulations. Communications in Statistics, (4), 104-123.

Hoerl, A. E., \& Kennard, R. W. (1970a). Ridge Regression: Biased Estimation for Non-orthogonal Problems. Technometrics, 12(55).

Hoerl, A. E., \& Kennard, R. W. (1970b). Ridge Regression: Applications for Non- orthogonal Problems. Technometrics, 12(69).

Hoerl, A. E., \& Kennard, R. W. (1976). Ridge regression: Iterative estimation of the biasing parameter. Commun. Statist. Theory Meth., 5(1), 77-88. http://dx.doi.org/10.1080/03610927608827333

Mal, C. Z., \& Dul, Y. L. (2014). Generalized shrunken type-GM estimator and its application. International Conference on Applied Sciences (ICAS2013).

Marquardt, D. W., \& Snee, R. D. (1975). Ridge regression in practice. Amer. Statist., (29), 3-19.

Martin, R. D. (2002). Robust Statistics with the S-Plus Robust Library and Financial Applications. 
Nikolai, K. (1992). An analysis of the Least Median of Squares regression problem. Proc. $10^{\text {th }}$.

Pariente, S., \& Welsch, R. E. (1977). Ridge and Robust Regression Using Parametric Linear Programming. Working Paper, MIT Alfred P Sloan School of Management.

Pfaffenberger, R. C., \& Dielman, T. E. (1984). A Modified Ridge Regression Estimator Using the Least Absolute Value Critrion in the Multiple Linear Regression Model (pp. 791-793) Proceedings of the American Institute for Decision Sciences, Toronto.

Pfaffenberger, R. C., \& Dielman, T. E. (1985). A Comparison of Robust Ridge Estimators. Proceedi of the American Statistical Association Business and Economic. Statistics Section. Las Vegas.

Pfaffenberger, R. C., \& Dielman, T. E. (1990). A comparison of regression estimators when both multicollinearity and outliers are present. Edit by: Arthur, Marcel Dekker, Inc. New York and Basel.

Rousseeuw, P. J. (1984). Least median of squares regression. J. Am. Stat. Assoc., 79, 871-880. http://dx.doi.org/10.1080/01621459.1984.10477105

Rousseeuw, P. J. (1985). Multivariate estimation with high breakdown point. Math. Stat. Appli., 283- 297.

Rousseeuw, P. J., \& Leroy, A. M. (1987). Robust Regression and Outlier Detection. Wiley-Interscience, New York. http://dx.doi.org/10.1002/0471725382

Siti, M. Z., Mohammad, S. Z., \& Muhammad, I. A. B. I. (2012). Weighted Ridge MM-Estimator in Robust Ridge Regression with Multicollinearity. Mathematical Models and Methods in Modern Science. Symp. Computational Statistics, 1, 471-476.

Venables, W. N., \& Ripley, B. D. (1999). Modern Applied Statistics with S-PLUS (4th ed.). Springer: New York. 167-176. http://dx.doi.org/10.1007/978-1-4757-3121-7

\section{Copyrights}

Copyright for this article is retained by the author(s), with first publication rights granted to the journal.

This is an open-access article distributed under the terms and conditions of the Creative Commons Attribution license (http://creativecommons.org/licenses/by/3.0/). 